\title{
Characteristics and Educational Advantages of Laboratory Automation in High School Chemistry
}

\author{
http://dx.doi.org/10.3991/ijoe.v7iS1.1787 \\ I. M. Verner and L. B. Revzin \\ Technion - Israel Institute of Technology, Haifa, Israel
}

\begin{abstract}
This paper presents a study of automation in the high school chemical inquiry based laboratory. Simple computer-controlled devices for automation of basic manual operations were constructed and applied in students' laboratory experiments together with the Fourier-Systems Inc. data collection and management systems. We examined characteristics of learning in the new automated laboratory environment and discussed educational outcomes.
\end{abstract}

Index Terms - chemical education, constructivist learning environment, high school, laboratory automation.

\section{INTRODUCTION}

In recent years, technology enhanced laboratory environments (TELE) have won growing popularity, especially in facilitating constructivist inquiry-based learning $[1,2]$. This trend significantly influences education in modern science laboratories. There is a lively debate about the educational objectives of practice in the technology enhanced laboratory and the ways to accomplish them [3]. Educators believe that new technologies can make laboratory experimentation more accessible and effective, providing significant advantages [4]:

- Reduce the need for physical contact with laboratory materials and devices.

- Foster self-learning.

- Allow sharing of experimental hardware.

- Provide computerized data management.

- Allow online remote experimentation.

- Enable effective use of laboratory resources.

Technology enhanced laboratories combine advantages of hands-on and computer simulation techniques, providing time-effective and focused experimentation, yet in real environments [5]. Implementation of TELE in chemistry education brings the additional benefit - preparing students for instrumental analysis in research and industrial laboratories [6].

Laboratory automation courses have been introduced in undergraduate and graduate chemistry curricula [7]. Recently the automation trend has involved high school chemistry laboratories. In Israel, the Advanced Chemistry discipline includes a unit called "Inquiry Based Laboratory" [8]. To study the unit, many school laboratories are equipped with computerized systems which provide automation of experimental data collection and analysis by means of sensors, data loggers and computers. Educa- tional studies indicated the positive effect of this practice on fostering students' higher order thinking skills [9].

It should, however, be noted, that while the computerized systems, currently used in the laboratory, provide automated data management, all manipulation operations are still performed manually. This causes difficulties in organizing laboratory studies that answer the safety, accuracy and timing requirements.

The goal of our on-going study is to develop, implement and evaluate a technology-rich environment for chemistry laboratory inquiries. In this environment high school students perform experiments using data management tools and computer controlled devices for automation of manual operations. We develop simple and affordable automated devices and involve students in constructing such devices and using them for laboratory experiments. In [10] we presented the first prototypes of our automated titrator and a computer controlled dispenser and the first experience of their use by students. Here we present the next step, at which we improved and developed automated devices and conducted systematic educational experiments in two different high schools.

\section{DeVelopment of Automation DeVices}

This section describes improvements and developments that we made in order to provide a broader automation and more effective use of the chemical laboratory.

\section{A. An Improved Automated Titrator}

The in-class experience of using the automated titrator prototype, described in [10], indicated some difficulties in operating the devise, which impaired accuracy of measurements. Particularly, in order to start titration synchronously with data collection and measurement, the student had to turn the stopcock and at the same moment to close the sensor circuit. To remove the problem, in the new prototype (Figure 1) the stopcock is replaced by a specially constructed operating device, in which pulling the handle down starts both the titrant delivery and the data collection.

\section{B. Peristaltic Pump}

Constant delivery rate in the abovementioned prototypes of the automated titrator was provided by a shopbought air pump. In these prototypes delivery rate cannot be computer controlled during the titration process, and therefore, the solution based on this pump cannot be used for constructing an automatic titrator. To solve this prob - 


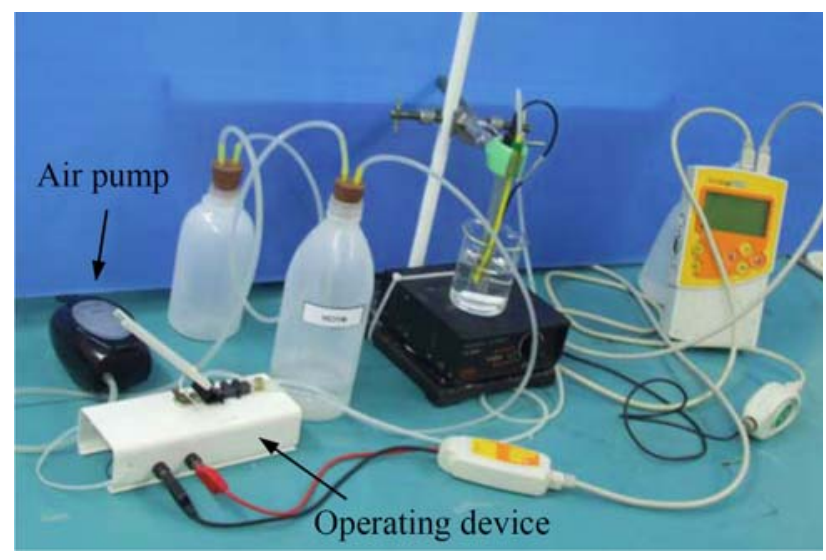

Figure 1. The improved automated titrator

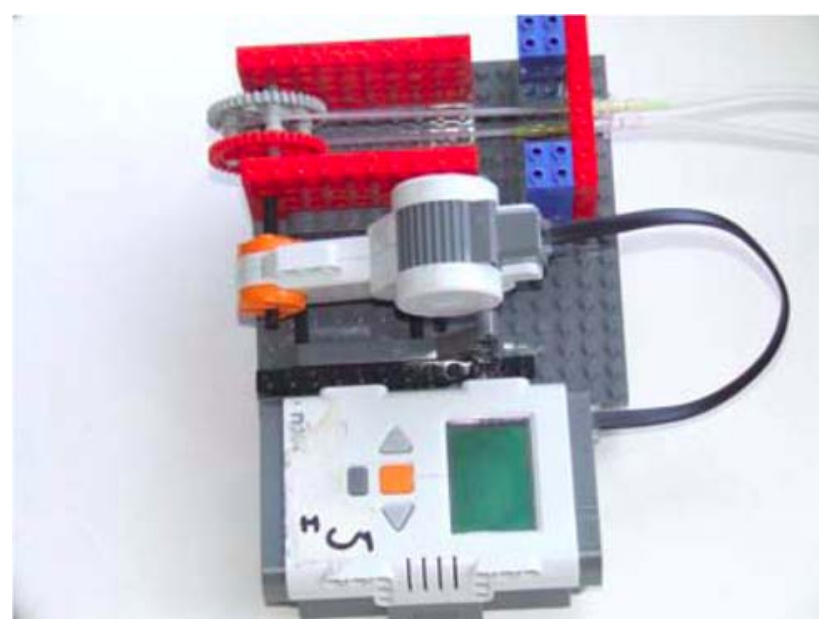

Figure 2. A peristaltic pump prototype

lem, we developed a Lego NXT based peristaltic pump prototypes presented in Figure 2.

In the pump a rotor moves four rollers which squeeze the flexible stretched tube, thus forcing the fluid, to be pumped, to move through the tube. Our experiments indicate that the pump accurately keeps the constant delivery rate proportional to the rotation speed which can be computer controlled.

\section{AUTOMATION SYSTEM ARCHITECTURE}

While in the conventional chemistry laboratory experiment the learner deals with the reactor and reagents directly, in the computerized and automated laboratory this connection is mediated by the technological system. This system includes devices for automated supply of chemical reagents to the reactor with tools for data management, providing integration of manipulation, measurement and data collection.

A possible architecture of such a system was proposed in [10]. Further teaching experience and research led us to understanding that the architecture of the computerized and automated chemistry laboratory should be based on the principles of the technology enhanced laboratory design [11-13]. Our vision for the laboratory system architecture is expressed by the diagram in Figure 3. In the system Web server provides collective access of users to laboratory facilities, Laboratory server manages the experimental process, Data logger collects experimental data from sensors, and Controller monitors automation devices.

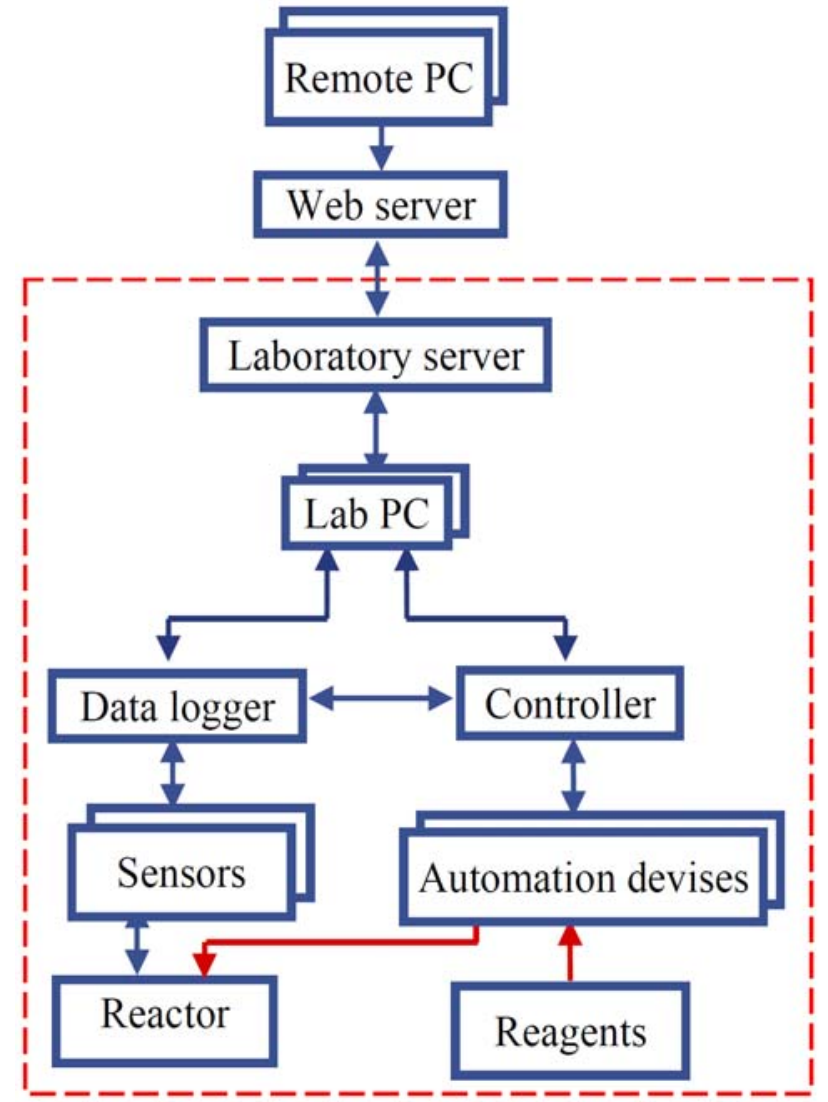

Figure 3. Laboratory system architecture

In this study we use the MultilogPro and USB Link data loggers connected to PC, or the NOVA 5000 computer with built-in data logger, all of Fourier Systems (www.fourier-sys.com). The sensors of Fourier and Vernier are applied (www.vernier.com).

The proposed architecture presents our vision of technology enhanced chemical laboratory, in which experimentation of the learner with reagents is mediated by technology and, therefore, can be carried out through remote operation. The red dotted line in Figure 3 outlines the configuration which was implemented in the reported study. Though remote operation is not yet realized, data management and lab operations are already automated, while the laboratory server provides in-class communication, and printing reports. Using a technology enhanced laboratory in chemical education offers several advantages [11]:

1. The technology enhanced laboratory provides efficient organization and intensification of experimental studies without increasing the space and personnel training laboratories.

2. Remote operation on one hand provides the opportunity to work with real materials and devices, and on the other hand significantly reduces the time of the real experiment.

3. Technology enhanced laboratory enables economical consumption of materials, makes the experiment more accurate and safe.

4. Through remote operation students can distantly access research and industrial laboratories, thus closing the gap between the chemistry class and modern chemical industry. 


\section{TEACHING EXPERIMENTS IN THE AUTOMATED LABORATORY}

\section{A. Comprehensive Urban HS Case 2009-2010}

We equipped the school chemistry laboratory with eight automated titrator prototypes described in [10]. Each titrator included the MultiLog Pro data logger, Fourier sensors, magnetic stirrer, and self-made constant delivery rate device. The automated titrators were used for experimentation of 11th and 12th grade students $(\mathrm{N}=101)$ studying chemistry at advanced level. The students experimentally investigated sedimentation processes using the conductivity sensor. At the beginning, they got familiar with the automated titration system. Four interested students got special training and served as teaching assistants, explaining the device's structure and operation rules to their peers. Then, all the students performed titrations in groups of 2-3. The workplace of each group was equipped with one of the automated titrators. The titrant delivery rate at each titrator was set different from the others, in order to provide unique results in each group.

The students determined the titrant's solution delivery rate and calculated precision. The measurements of weight and volume were made by analytical balances and graduated cylinders. Their accuracy was compared, while the means and standard deviations of errors were calculated using Excel - the technique that was new to the students. The second experiment determined the concentration of titrant by titrating aliquots of standard solution. Then the students conducted two their own studies using the automated titrators. They formulated research questions, planned related experiments, prepared an order for the needed equipment and reagents, performed the experiments by teacher's permission, processed the received data, discussed the results in groups, and wrote final reports.

Students' Reflections on the Automated Laboratory

- "The purpose of the lab is to teach us for future "real world". Therefore, equipment should be more modern and it seems like we'll use the devices in the future."

- "Automation devices cut down the labor process, helped to the research..."

- "Use of automated instruments has contributed to understanding the experimental material"

- "There is no problem with them and can be used"

- "Using automated instruments helped in many implementations, shortened the time and increased the efficiency of the process of experiment"

- "Needs to improve, sometimes not working well"

- "It was hard to understand, only after a detailed explanation of the teacher could use"

- "Automation makes the experiment more difficult to perform "

- "Only facilitated the implementation of designs"

- "Contributed to the accurate results for all test and add to ease processes."

- "Helped a lot, sometimes got stuck and made problems."

\section{B. Comprehensive Urban HS Case 2010-2011}

In this case we equipped the laboratory of the same high school with modified automated titrators described in Section II.A. They were used throughout the school year by two groups of 11th graders $(\mathrm{N}=50)$ and two groups of 12 th graders $(\mathrm{N}=42)$. The laboratory course started with a two-hour lesson, at which during the first hour the acid base titration by means of the $\mathrm{pH}$ sensor, construction and operation of the automated titrator were introduced. At the second hour the students exercised in performing titrations. The modified titrators, installed in the laboratory this year, worked reliably and satisfied the experimentation needs of the whole class (12-15 titrations per each device at each lesson).

As the modified titrator is easy for use, the students operated it without assistance. It was enough to use only four modified titrators instead of eight previous ones used in the past year. The students worked in groups and conducted seven experiments, two of them were the same as in the past year and five others were new and open-ended.

The effectiveness of the experimentation was achieved, to a large extent, due to the modified titrator. By opinion of students, teachers and the laborant, the modified titratorit has the following advantages comparative to the first prototype:

- One button based turn on/off of the titration system instead of operating the stopcock.

- Changing a titrant solution takes only 2-3 minutes, significantly less than in the previous version.

- Short instruction for using the titrator.

As a result of these "technical" advanteges, the style of learning in the laboratory significuntly changed. From our observations, the priciple changes were:

1) More attention and time to planning and analysis of experiments.

The students took seriously planning and carrying out their experiments, and felt personal responsibility for its results. They were self directed and ready to repeat the experiment if, for their opinion, its result was not accurate. In particular, one of the first laboratory exercises was to experimentally determine concentration of standard sodium hydroxide aqueous solution by titration with $1 \mathrm{M}$ chlorine acid solution. The sodium hydroxide concentration was calculated based on the titration results. Then, the teacher gave the students the exact value of the standard solution concentration and the students compared it with their results. For all the groups, except two, the error did not exceed $0.5 \%$. Though the students' grades did not depend on the achieved accuracy, the students from these two groups by their own repeated the experiment and achieved an accurate result. Many students expressed satisfaction of the laboratory practice and noted that they felt beauty of the experiment and elegance of its implementation.

2) More creativity and initiative in laboratory inquiries

The experiments had become more scientific with deeper research questions. The students were highly motivated to search literature and web sources for theoretical and practical foundations of the experiments. For this purpose they used the collection of chemistry books available in the laboratory. As the lab computers were used for data collection and analysis, many students brought own lap- 
tops for operative web search on the topics of their inquiry. They initiated discussions within and among the groups and shared knowledge and results. For instance, the students developed procedures for experimental determination of free acids in olive oil by back titration, for evaluating the degree of turbidity of solutions using the colorimeter sensor. The students developed, constructed and experimentally examined (in their free time) three different devices for measuring density of liquids, based on their own ideas. The students developed mathematical models and used them to analyze performance of each device. One of the students, who studied advanced chemistry and advanced robotics courses, got a scholarship for first degree studies in chemical engineering granted by the TAMI R\&D Center of Industrial Chemistry (based on recommendation of the authors).

\section{3) 3. More efficient communication and partnership}

From our observation, the students more carefully selected partners for teamwork. "Weaker" students tended to team with knowledgeable students, while the latter apt to work with "equal" partners. Theoretically inclined students were interested to collaborate with experimentally inclined teammates. The "strong" teams excelled in productive discussions and significant contributions of all its members. We did not meddle in team forming but tried to adapt guidance to address specific needs of each team. The striking change in interaction with the teachers is that student teams involve them in discussions at all stages of the experiment, from the plan and set-up to the analysis and conceptualization of results. We note, that collective use of automation systems led to consolidation among the students and shaping the community of experimental inquiry.

\section{Vocational HS Case 2009-2011}

We introduced chemistry studies in an unprivileged vocational high school for students majoring in mechanical technology. The school is located in the Haifa's surrounding zone of chemical industry where there is a lack of technical personnel. Therefore, the school sought to prepare its students for future work in chemical plants.

Most of the students in this school are regarded as "at risk" and, therefore, involving them in chemistry studies was a challenge. Traditional methods of teaching school chemistry were not appropriate for this group, because the students did not have prerequisite knowledge and skills for learning science. In search of possible solutions, the school chose our approach based on connecting the studies of chemistry and automation technology through collaboration of the chemistry and robotics teachers. This approach was implemented through collaboration of the school with the TAMI Institute, which provided its chemistry laboratory, the Fourier Systems Company, which granted its data loggers and sensors, and our Technion group, which developed and implemented the chemistry course. The collaborative project was conducted under supervision of Israeli Ministry of Education.

Twelve students (grades 11 and 12) studied the chemistry course in 2009-2010. It started from laboratory lessons, at which the students learned the NOVA 5000 computer and sensors and used them to conduct simple experiments, such as determining the ice melting, water boiling and water freezing points. In parallel, at the robotics lessons

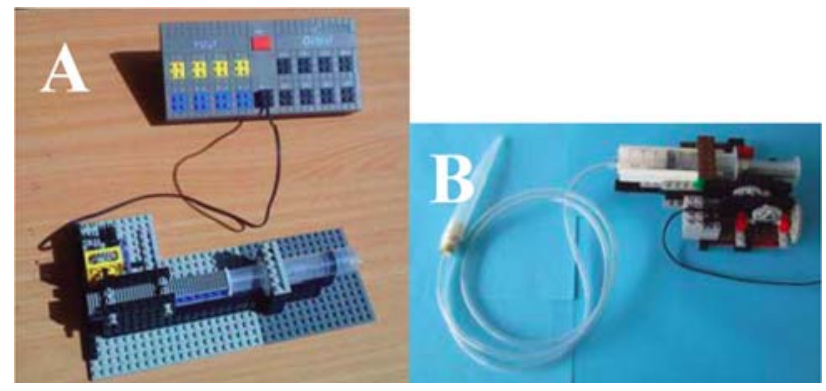

Figure 4. Computer controlled syringe pumps used: A. For studying ideal gas laws; B. In the automated dispenser.

the students constructed mechanical models of a syringe pump (Figure 4).

At the second step the students made experiments in the chemistry laboratory in order to examine characteristics and usability of the constructed mechanical models. In particular, the students tested and improved their models by applying them together with NOVA 5000 and sensors for inquiry into gas laws. From these experiments the students got familiar with physical parameters of gas (temperature, volume, mass, and pressure) and acquired basic skills of data analysis (interpreting functions, graphs and tables). Following the inquiry experience, the students became motivated to learn chemistry.

After studying the atomic structure, the periodic table of the elements and other basic concepts, the students came to the third step in connecting chemistry and automation. This step was offered to the students who took the whole 2-year course. It started at the end of the first year, continued during the second year, and finished by matriculation exams in both automation and chemistry. At this step the students in pairs performed project assignments, which required developing a device for automation of a certain laboratory function. The project was performed in the chemistry laboratory. The students developed computer controlled automation devices and applied them for inquiry into chemical processes. One of the devices, an automated titrator, is presented in Figure 5. The device includes a syringe pump, a mixer, a sensor's holder, and a lifting mechanism - all driven by two connected PicoCricket controllers (www.picocricket.com).

A significant change of students' behavior has been observed over their two-year participation in the course. At the beginning of the course we encountered discipline and communication problems, as well as lack of learning motivation. During the course the students have become more responsible, communicative, and interested in the subject, especially in laboratory experimentation. During the second year there were no behavior problems and violations of the laboratory safety rules. Students by their own prepared experimental setups, performed experiments and maintained the workplaces without any technical assistance. While every lesson in the first year of the course was attended by the overseer, it was no longer needed in the second year. Moreover, 12th graders served as laboratory assistants for the 11th graders who started to study the course.

Three control tasks were assigned to the students at the end of the course in order to assess their learning outcomes. The first task was to mentor the 11th grade students, who started the course, about the proper and safe 


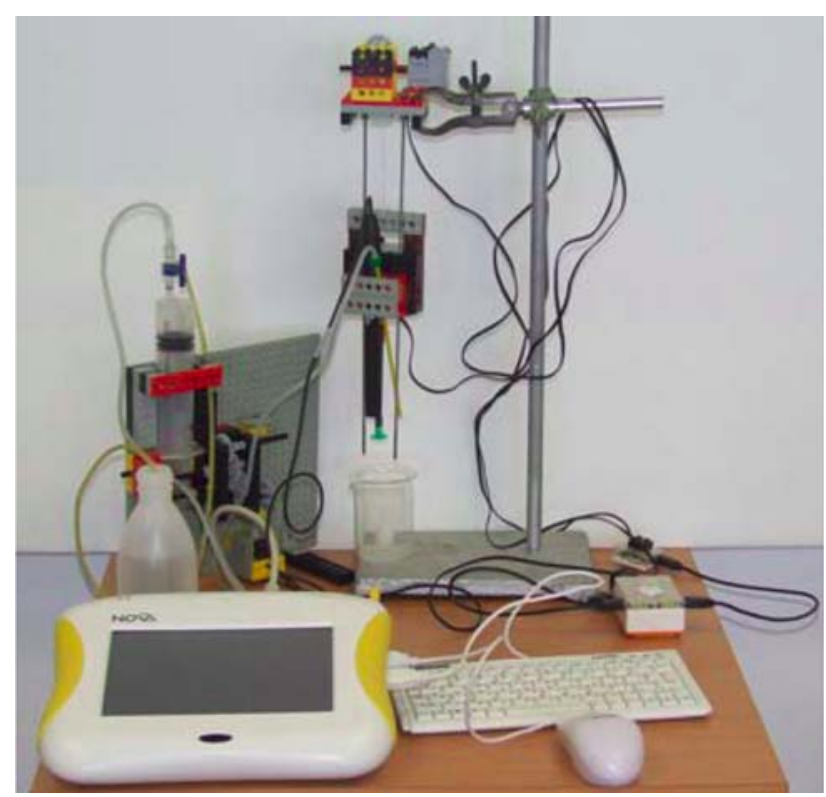

Figure 5. Automated titrator project

use of tools and materials in the chemistry laboratory. In the second task the students were required to conduct an unfamiliar experiment in accordance with the manual book instructions. In the third task each of the students answered the questionnaire about his self-efficacy in the chemistry laboratory work skills.

It is important to note that all the students succeeded in the control tasks. As mentors, they really helped the teacher by promoting safe and responsible behavior in the laboratory. The teacher's diary includes numerous examples, when the 12th graders found mistakes of the 11th graders in the experiment and showed proper way to perform it. Regarding the unfamiliar experiment, the students by their own successfully conducted it, got and saved the data as graphic files. However, because of the lack of theoretical background in the subject, they had difficulties in interpreting results of the experiment.

All the students expressed high self-efficacy beliefs. They are confident about their ability of proper and safe laboratory experimentation, including the competences of connecting, testing and calibrating sensors and automation devices. The students believe that after some workplace training they will be able to work as chemistry laboratory assistants. They expressed interest to study industrial chemistry in the technical college.

\section{V.CONCLUSION}

Our experience of creating simple affordable automated devices, their integration with a data logging system and teaching automated laboratories indicates the considerable potential of this technology for improving experiential chemistry education in high schools. Application of the developed devices enables to save time, spent for performing manual operations, and focus the laboratory on inquiry activities. It also enables to raise the accuracy and safety of the experiments by eliminating errors and incidents related to manual operations.

From the experience, development of simple and affordable automated devices and implementation of TELE in school chemistry laboratories is a challenging problem. A promising approach is through creating centralized interschool laboratories with opportunities of local and remote experimentation. In the paper we presented a possible architecture for such a system and described how the automated devices were improved, based on their practical use. Analysis of the laboratory practice led us to understanding specific requirements to school laboratory automation devices:

- Operational control of the process.

- Simplicity and reliability of operation.

- Options for individual and collective use.

- Easy construction from low-cost parts.

- The device should not interfere with the observation of the process.

Creating effective automation devices is possible only if it is followed by the study of the experiential learning processes. In the paper we consider two case studies: one in the comprehensive high school and the other in the vocational high school. In both cases we have seen that the automated laboratory environment can be effectively used. For the comprehensive HS the main effectiveness factors were a sharp increase in experimental productivity and, as a result, dramatic expansion of inquiry opportunities. As for the vocational HS, implementation of the proposed approach enabled the real opportunity of studying the chemistry science to students, to whom the conventional methods of science education were inappropriate.

Further development and research are needed in order to open and investigate the opportunities of technologyenhanced learning environments and their impact on chemistry education in schools.

\section{ACKNOWLEDGMENT}

The authors thank Dr. Nitsa Barnea for interest and encouragement in this work.

\section{REFERENCES}

[1] F. Wang, and M. J. Hannafin, "Design-based research and technology-enhanced learning environments," Educational Technology Research and Development, vol. 53, no. 4, pp. 5-23, 2005.

[2] M. C. Kim, M. J. Hannafin, and L. A. Bryan, "Technologyenhanced inquiry tools in science education: An emerging pedagogical framework for classroom practice," Science Education, vol. 91, pp. 1010 - 1030, 2007.

[3] L. D. Feisel and G. D. Peterson, "A colloquy on learning objectives for engineering education laboratories," in Proceedings of the 2002 American Society for Engineering Education Annual Conference and Exposition, 2002.

[4] I. Mougharbel, A. El-Hajj, H. Artail, and C. Riman, "Remote lab experiments models: A comparative study," International Journal of Engineering Education, vol. 22, no 4, pp. 849-857, 2006.

[5] J. V. Nickerson, J. E. Corter, S. K. Esche, and C. Chassapis, "A model for evaluating the effectiveness of remote engineering laboratories and simulations in education," Computers and Education, vol. 49, pp. 708-725, 2007. http://dx.doi.org/10.1016/j.comp edu.2005.11.019

[6] D. Kennepohl, J. Baran, M. Connors, K. Quigley, and R. Currie, "Remote access to instrumental analysis for distance education in science," International Review of Research in Open and Distance Learning, vol. 6, no. 3, pp. 1-14, 2005.

[7] J. D. Sterling, "Laboratory automation curriculum at Keck Graduate Institute," Journal of the Association for Laboratory Automation, vol. 9, no. 5, pp. 331-335, 2004. http://dx.doi.org/ 10.1016/j.jala.2004.07.005

[8] N. Barnea, Y.J. Dori and A. Hofstein, "Development and implementation of inquiry-based and computerized-based laboratories: 
reforming high school chemistry in Israel," Chemistry Education Research and Practice, vol. 11, pp. 218-228, 2010.

[9] Z. Kaberman, and Y. J. Dori, "Question posing, inquiry, and modeling skills of chemistry students in the case-based computerized laboratory enviroment," International Journal of Science and Mathematics Education, vol. 7, pp. 597-625, 2009. http://dx.doi.org/10.1007/s10763-007-9118-3

[10] I. M. Verner, and L. B. Revzin, "Automation of manual operations in a high school chemistry laboratory: Development and implementation," Chemical Educator, vol. 15, pp. 141-145, 2010.

[11] J. Baran, R. Currie, and D. Kennepohl, "Remote instrumentation for the teaching laboratory," Journal of Chemical Education, vol. 81, no. 12, pp. 1814-1816, 2004. http://dx.doi.org/10.1021/ ed081p1814

[12] Y. Yan, Y. Liang, X. Du, H. Saliah-Hassane, and A. Ghorbani, "Putting labs online with web services," IT Pro, vol. 8, no. 2, pp. 27-34. 2006. http://dx.doi.org/10.1109/MITP.2006.45
[13] O. Naef, "Real laboratory, virtual laboratory or remote laboratory: What is the most efficient way?" iJOE, vol. 2, no. 3, 2006, http://www.online-journals.org/index.php/i-joe/issue/view/36.

\section{AUTHORS}

I. M. Verner is with the Department of Education in Technology and Science, Technion - Israel Institute of Technology, Haifa, 32000 Israel (e-mail: ttrigor@technion.ac.il).

L. B. Revzin is with the Department of Education in Technology and Science, Technion - Israel Institute of Technology, Haifa, 32000 Israel (e-mail: leonidre.ih@gmail.com).

This article is an extended version of a paper presented at the International Conference EDUCON2011, held in April 2011 at PSUT, in Amman, Jordan. Received 08 August 2011. Published as resubmitted by the authors September $2^{\text {nd }}, 2011$. 